\title{
Stabilized micelles as delivery vehicles for paclitaxel
}

Krassimira Yoncheva ${ }^{1}$, Patricia Calleja ${ }^{2}$, Maite Agüeros ${ }^{2}$, Petar Petrov $^{3}$, Ivanka Miladinova ${ }^{1}$, Christo Tsvetanov ${ }^{3}$, Juan M. Irache ${ }^{2}$

${ }^{1}$ Department of Pharmaceutical Technology and Biopharmacy, Faculty of Pharmacy, 2 Dunav Str., 1000 Sofia, Bulgaria

${ }^{2}$ Department of Pharmacy and Pharmaceutical Technology, University of Navarra, 31080 Pamplona, Spain

${ }^{3}$ Institute of Polymers, Bulgarian Academy of Sciences, 103 Akad. G. Bonchev Str., 1113 Sofia, Bulgaria

Corresponding author:

K. Yoncheva, $\mathrm{PhD}$

Dept. Pharmaceutical Technology and Biopharmaceutics

Faculty of Pharmacy

2 Dunav Str.

1000 Sofia

Bulgaria

Tel. (+359)29236527

Fax (+359)29879874

e-mail: krassi.yoncheva@gmail.com 


\begin{abstract}
Paclitaxel is an antineoplastic drug used against a variety of tumors, but its low aqueous solubility and active removal caused by P-glycoprotein in the intestinal cells hinder its oral administration. In our study, new type of stabilized Pluronic micelles were developed and evaluated as carriers for paclitaxel delivery via oral or intravenous route. The pre-stabilized micelles were loaded with paclitaxel by simple solvent/evaporation technique achieving high encapsulation efficiency of $70 \%$. Gastrointestinal transit of the developed micelles was evaluated by oral administration of rhodamine-labeled micelles in rats. Our results showed prolonged gastrointestinal residence of the marker encapsulated into micelles, compared to a solution containing free marker. Further, the oral administration of micelles in mice showed high area under curve of micellar paclitaxel (similar to the area of i.v. Taxol ${ }^{\circledR}$ ), longer mean residence time (9-times longer than i.v. Taxol ${ }^{\circledR}$ ) and high distribution volume (2-fold higher than i.v. Taxol ${ }^{\circledR}$ ) indicating an efficient oral absorption of paclitaxel delivered by micelles. Intravenous administration of micelles also showed a significant improvement of pharmacokinetic parameters of micellar paclitaxel vs. Taxol ${ }^{\circledR}$, in particular higher area under curve (1.2-fold), 5-times longer mean residence time and lower clearance, indicating longer systemic circulation of the micelles.
\end{abstract}

Keywords: stabilized micelles; paclitaxel; Pluronic; gastrointestinal transit; oral absorption 


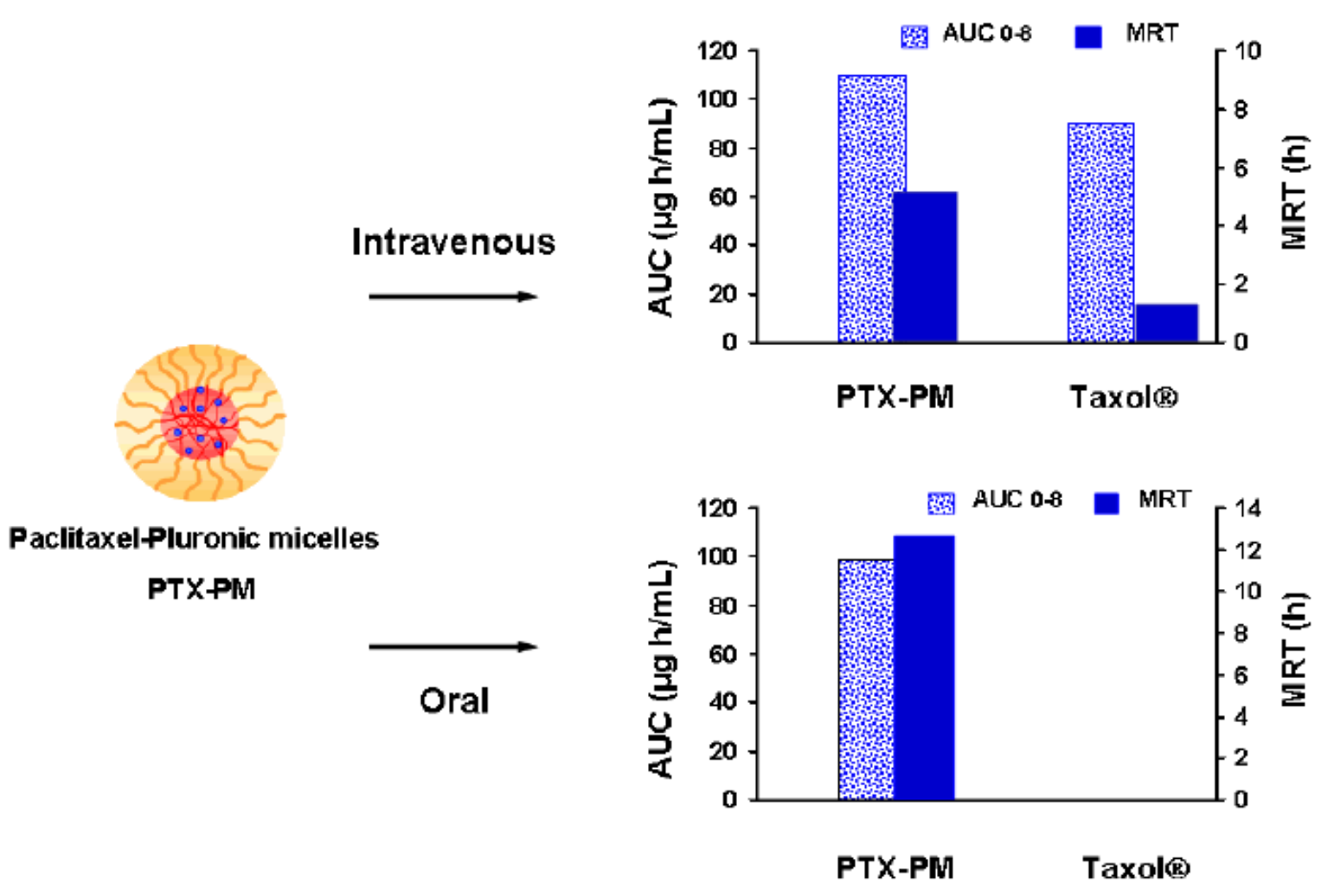

\section{Graphical abstract}

Schematic presentation describing the improved pharmacokinetic parameters achieved by administration of paclitaxel-loaded stabilized Pluronic micelles (PTX-PM). For comparison, the same parameters calculated after administration of paclitaxel in the form of commercial solution $\left(\operatorname{Taxol}^{\circledR}\right)$. 


\section{Introduction}

Polymeric micelles present a great potential as drug delivery systems for compounds that are hydrophobic and exhibit poor bioavailability. The unique core-shell structure of micelles enables incorporation of poorly soluble drugs into the inner hydrophobic core thus improving their stability and bioavailability. The hydrophilic shell plays an important role for the in vivo behavior of the micelles, in particular their steric stabilization and ability to interact with the cells. According to some references polymeric micelles could facilitate drug transport by fluid phase endocytosis as well as to provide receptor mediated transport by functionalization of the shell (Torchilin, 2004; Mathot et al., 2007).

The micelles of poly(ethylene oxide)-b-poly-(propylene oxide)-b-poly(ethylene oxide) (PEO-PPO-PEO) amphiphilic triblock copolymers are evaluated as drug and gene delivery systems, as well as in diagnostic imaging as carriers for various contrasting agents (Kabanov et al., 2002). PEO-PPO-PEO micelles can be prepared from commercially available copolymers (Pluronics) and combine several attractive properties, including resistance of the PEO shell to protein adsorption, ability of the temperature responsive PPO core to solubilize water-insoluble compounds and availability of hydroxyl groups to which receptor-specific ligands can be attached (Alexandridis and Hatton, 1995). Since the formation of PEO-PPOPEO micelles is a dynamic phenomenon, one possible drawback may be associated with micellar disaggregation upon dilution at physiological conditions. Rapoport and co-workers proposed different strategies for stabilization of PEO-PPO-PEO micelles, in particular radical cross-linking of micelle core using benzoyl peroxide as radical initiator, introduction of vegetable oil aiming to increase hydrophobic interactions within the core, or formation of hydrogel inside the micelle core (Plurogel®) (Rapoport, 1999). More recently, an efficient method for the stabilization of PEO-PPO-PEO micelles by UV-induced formation of an interpenetrating network of poly(pentaerythritol tetraacrylate), in which the polyether chains 
were physically entrapped, has been reported (Petrov et al., 2006). The stabilized polymeric micelles (SPM) resisted changes in concentration and solvent and maintained their structure and size even when ultrasound irradiated at $20 \mathrm{kHz}$.

Paclitaxel (PTX) is an antineoplastic drug successfully used against a variety of tumors including ovarian, breast and non-small cell lung tumors (Gregory and DeLisa, 1993; Chen et al., 2003). In clinics, paclitaxel is intravenously administered as infusion (i.e. Taxol®). Due to its limited aqueous solubility $(0.3 \mu \mathrm{g} / \mathrm{ml})$, the commercial injectable formulations use a mixture of Cremophor EL and ethanol (1:1 v/v) (Singla et al., 2002). Apart from other problems, the presence of Cremophor EL results in hypersensitivity reactions, nephrotoxicity, and neurotoxicity (Onetto et al., 1993). In addition, this pharmaceutical excipient can also modify the pharmacokinetics of PTX (Sparreboom et al., 1999). To overcome these problems, several alternative pharmaceutical carriers have been developed for PTX delivery including liposomes (Wang et al., 2010; Zhao et al., 2011), polymeric micelles (Hamaguchi et al., 2005; Forrest at el., 2008) and nanoparticles (Koziara et al., 2006; Win and Feng, 2006). In the last years, different paclitaxel formulations have been clinically studied such as Opaxio ${ }^{\circledR}$ (formerly Xyotax ${ }^{\circledR}$ ) and Abraxane ${ }^{\circledR}$. The former is a polymer conjugate between paclitaxel and poly(glutamate) (Boddy et al., 2005) whereas the latter, which was recently commercialised, is based on the incorporation of paclitaxel in human serum albumin nanoparticles (Roy et al., 2009).

On the other hand, a number of different attempts have been performed to develop oral formulations of paclitaxel. Nevertheless, apart from the low aqueous solubility, oral treatment with paclitaxel is hampered by the fact that the drug is substrate of the P-glycoprotein and cytocrome P-450 (Letchford et al., 2009). As a result the oral bioavailability of paclitaxel is extremely low (Malingre et al., 2001). In the last years, different approaches have been proposed to solve this problem including the co-administration of pharmaceutical excipients 
with capability to disturb the effect of intestinal P-gp and/or cytocrome P450. In this context, the combination between cyclodextrins and poly(anhydride) nanoparticles yielded oral bioavailability up to $85 \%$ in rat. In this approach, the cyclodextrin permits to promote the encapsulation of the lipophilic drug into the nanoparticles and to inhibit the activity of the Pgp, whereas the function of poly(anhydride) nanoparticles is to develop bioadhesive interactions within the gastrointestinal mucosa and control the release of their content (Agüeros et al., 2010).

The aim of this work was to prepare, characterize and evaluate the pharmacokinetics of paclitaxel incorporated in stabilized Pluronic micelles. The stabilization of micelles by crosslinking of their core was performed aiming to prevent disaggregation of micelles upon dilution in physiological fluids. On the other hand, drug encapsulation in micelles can diminish drug extravasation to normal tissues and provide a passive drug targeting to tumors via the enhanced permeability and retention effect (EPR) (Maeda et al., 2000). This increased permeability is favorised by the prolonged circulation of micelles and lack of renal clearance (Han et al., 2006; Batrakova et al., 2008). On the other hand, Pluronic copolymers (as other pharmaceutical excipients) may inhibit the activity of drug efflux transporters such as P-gp, MRPs and BCRP (Kabanov et al., 2002; Thomas et al., 2003; Huang et al., 2008). Thus, the combination of these properties with the fact that the shell of polymeric micelles could establish interactions with the cells may be an adequate strategy to (i) increase the oral bioavailability and/or (ii) promote the efficacy of the loaded drug.

\section{Materials and methods}

\subsection{Materials}

$\mathrm{PEO}_{43} \mathrm{PPO}_{15} \mathrm{PEO}_{43}$ (Pluronic F-38; MW 4700 gmol $^{-1}$ ) were kindly donated by BASF Pentaerythritol tetraacrylate (PETA) and rhodamine B were purchased from Aldrich. 
Paclitaxel (USP 26 grade $>99.5 \%$ ) was supplied by 21CEC (London, United Kingdom). Docetaxel $\left(\right.$ Taxotere $^{\circledR}$ ) was provided by Sanofi-Aventis (Paris, France). Taxol ${ }^{\circledR}$ was purchased from Bristol-Myers Squibb (New York, USA). All other solvents and reagents were of analytical grade.

\subsection{Preparation of stabilized Pluronic micelles (PM)}

$\mathrm{PEO}_{43} \mathrm{PPO}_{15} \mathrm{PEO}_{43}(5 \mathrm{~g})$ was dissolved in $250 \mathrm{ml}$ double distilled water in a glass vessel under stirring. Acetone solution of PETA $(0.05 \mathrm{~g} / \mathrm{ml})$ was added and the temperature of the solution was increased to $60{ }^{\circ} \mathrm{C}$. Argon was bubbled through the solution for $45 \mathrm{~min}$, followed by irradiation with a full spectrum UV light (TQ 150 Original Hanau high-pressure $150 \mathrm{~W}$ mercury lamp provided with a quartz tube and a cooling quartz jacket) for $45 \mathrm{~min}$. The stabilized polymeric micelles were purified by dialysis against water using a cellulose membrane (Sigma, cutoff 12,000 gmol $^{-1}$ ) for 14 days. Finally, the micelles dispersion was frozen and subsequently lyophilized.

\subsection{Loading of pre-stabilized Pluronic micelles with rhodamine (R-PM)}

The pre-stabilized micelles were fluorescently labelled with rhodamine B by solvent evaporation technique. For this purpose, $10 \mathrm{ml}$ ethanol solution of rhodamine (concentration $0.1 \mathrm{mg} / \mathrm{ml}, 0.15 \mathrm{mg} / \mathrm{ml}$ or $0.3 \mathrm{mg} / \mathrm{ml}$ ) was added to $10 \mathrm{ml}$ of aqueous dispersion of the stabilized micelles $(3 \mathrm{mg} / \mathrm{ml})$ and the compounds were incubated for $24 \mathrm{~h}$. The ethanol was evaporated under reduced pressure (Buchi-144, Switzerland) and dialysis of the aqueous dispersion towards distilled water (Sigma, cutoff $12,000 \mathrm{~g} \mathrm{~mol}^{-1}$ ) was performed in order to eliminate the free rhodamine. Finally, the purified aqueous dispersion containing rhodamine loaded micelles was lyophilized (Genesis 12EL, Virtis, USA) using sucrose as cryoprotector $(5 \% \mathrm{w} / \mathrm{v})$. 


\subsection{Loading of pre-stabilized Pluronic micelles with paclitaxel (PTX-PM)}

Ten $\mathrm{ml}$ of ethanol solution of paclitaxel (concentration $0.1 \mathrm{mg} / \mathrm{ml}, 0.15 \mathrm{mg} / \mathrm{ml}$ or 0.3 $\mathrm{mg} / \mathrm{ml}$ ) was added to $10 \mathrm{ml}$ of aqueous dispersion of the pre-stabilized micelles $(3 \mathrm{mg} / \mathrm{ml})$ and the compounds were incubated for $24 \mathrm{~h}$. The ethanol was evaporated under reduced pressure (Buchi-144, Switzerland) and the resulted micelle dispersion was filtered $(0.22 \mu \mathrm{m})$. The filter was rinsed with ethanol and these fractions were determined for non-encapsulated paclitaxel. The aqueous dispersion containing paclitaxel loaded micelles was lyophilized (Genesis 12EL, Virtis, USA) using sucrose as cryoprotector (5\% w/v).

\subsection{Characterisation of the micelles}

Size and zeta-potential were determined by photon correlation spectroscopy and electrophoretic laser doppler anemometry using a Zetamaster analyzer (Malvern Instruments, UK). Samples were dispersed in either distilled water or saline $(0.154 \mathrm{M}$ and $0.308 \mathrm{M} \mathrm{NaCl}$ solution) and measured at $25^{\circ} \mathrm{C}$ with a scattering angle of $90^{\circ}$.

\subsection{Determination of drug loading}

The amount of rhodamine incorporated into the R-PM was determined by spectrofluorimetry at wavelength $\lambda_{\mathrm{ex}}=540 \mathrm{~nm}$ and $\lambda_{\mathrm{em}}=580 \mathrm{~nm}$ (GENios, Austria). The marker loading was calculated as a difference between its initial concentration and the concentration found in the aqueous fractions collected after the dialysis procedure. For the calculations standard curve of rhodamine B in distilled water was prepared in the concentration range of $0.2-4.0 \mu \mathrm{g} / \mathrm{ml}(\mathrm{r}>0.9978)$.

The amount of paclitaxel incorporated into the PTX-PM was calculated as a difference between its initial concentration and the concentration found in the ethanol rinsing fractions. Paclitaxel was determined by HPLC method, in particular the chromatographic system was an 
Agillent 1100 series (Waldbornn, Germany), coupled with a UV diode array detection system (25). Data were analyzed using the Chemstation G2171 program (B.01.03). The separation of PTX was carried out at $30^{\circ} \mathrm{C}$ on a reversed-phase $150 \times 3 \mathrm{~mm} \mathrm{C} 18$ Phenomenex Gemini column (particle size $5 \mu \mathrm{m}$ ). The mobile phase, pumped at $0.5 \mathrm{ml} / \mathrm{min}$, was $50: 50$ acetonitrile - phosphate buffer (0.02 M, pH=2.0) and effluent was monitored with UV detection at 228 $\mathrm{nm}$. For the calculations standard curve of paclitaxel was prepared in the concentration range of $1.25-80.0 \mu \mathrm{g} / \mathrm{ml}(\mathrm{r}>0.9991)$. The limit of quantification was calculated as $40 \mathrm{ng} / \mathrm{ml}$ with a relative standard deviation of $5.2 \%$.

The equations used for calculation of theoretical drug loading, actual drug loading and encapsulation efficiency are given bellow:

$$
\begin{gathered}
\text { Theoretical drug loading }(\%)=\frac{\text { Weight of drug initially added }}{\text { Weights of drug and stabilized micelles }} \times 100 \\
\text { Actual drug loading }(\%)=\frac{\text { Weight of drug incorporated into micelles }}{\text { Weight of drug loaded micelles }} \times 100 \\
\text { Encapsulation efficiency }(\%)=\frac{\text { Actual drug loading }}{\text { Theoretical drug loading }} \times 100
\end{gathered}
$$

\subsection{Distribution of R-PM within the gut}

The study was performed in compliance with the regulations of the responsible committee of the University of Navarra in line with the European legislation on animal experiments (86/609/EU). Rhodamine B labelled micelles were administered in the form of aqueous suspensions $(10 \mathrm{mg} / \mathrm{ml})$ to fasted Wistar rats orally. The animals were sacrificed by cervical dislocation at 1,3 and $8 \mathrm{~h}$ post administration. The abdominal cavity was opened and GIT was removed and divided into three regions: stomach, small intestine and caecum. Each segment was opened lengthwise along the mesentery and rinsed with phosphate saline buffer 
$(\mathrm{pH} 7.4)$ in order to eliminate the lumen contents. Further, each segment was cut into portions of $2 \mathrm{~cm}$ length and digested in $1 \mathrm{ml}$ of $3 \mathrm{~N} \mathrm{NaOH}$ for $24 \mathrm{~h}$ (Arbos et al., 2002). Rhodamine was extracted with $2 \mathrm{ml}$ methanol, vortexed for $1 \mathrm{~min}$ and centrifuged at $4000 \mathrm{rpm}$ for 10 min. Aliquots $(1 \mathrm{ml})$ of the supernatants were assayed for rhodamine by spectrofluorimetry (GENios, Austria) to estimate the fraction of the micelles within gastrointestinal mucosa. Calculations were made using standard curves of rhodamine prepared by addition of rhodamine solutions in $3 \mathrm{~N} \mathrm{NaOH}(0.5-10 \mu \mathrm{g} / \mathrm{ml})$ to tissue segments following the same treatment steps.

Control rhodamine solution $(119 \mu \mathrm{g} / \mathrm{ml})$ was administered to rats and the presence of rhodamine within gastrointestinal mucosa was determined following the same procedures as above.

\subsection{Pharmacokinetic study in mice}

Animal experiments were performed in compliance with regulations of the responsible Ethical Committee of the University of Navarra (protocol number 076-06) in strict accordance with the European legislation in animal experiments. Female C57BL/6J mice (average weight 20 g) (Harlan, Spain) were housed under normal conditions with free access to food and water. The animals were placed in metabolic cages and fasted overnight to prevent corpophagia but allowing free access to water.

For the pharmacokinetic study, the mice were divided at random into 4 groups $(n=5)$. The first two groups received orally a single dose (10 $\mathrm{mg}$ paclitaxel $/ \mathrm{kg})$ of either (i) commercial Taxol $^{\circledR}$ or (ii) paclitaxel-loaded micelles (PTX-PM). Besides, Taxol ${ }^{\circledR}$ and PTX-PM were also intravenously administered to the other two groups of mice at the same dose $(10 \mathrm{mg} / \mathrm{kg})$.

All the formulations were administered dispersed or dissolved in $1 \mathrm{ml}$ of water (oral) or saline (intravenous). Blood samples of $200 \mu \mathrm{l}$ were collected at different times in tubes 
containing EDTA (Microvette ${ }^{\circledR}$ EDTA tripotassium salt; Sarstedt, Numbrecht, Germany). The volemia was recovered via intraperitoneal injection of an equal volume of normal saline solution preheated at body temperature. Blood samples were centrifuged for 10 min at 10,000 rpm and the supernatant plasma fractions were stored at $-80^{\circ} \mathrm{C}$ until HPLC analysis.

\subsection{HPLC quantification of paclitaxel in plasma samples}

The amount of paclitaxel was determined in plasma by HPLC as described above. Calibration curves were used for the conversion of the PTX/DCX chromatographic area to the concentration. Calibrator and quality control samples were prepared by adding appropriate volumes of standard PTX ethanol solution to drug free plasma. Calibration curves were designed over the range of $40-3200 \mathrm{ng} / \mathrm{ml}\left(\mathrm{r}^{2}>0,999\right)$. An aliquot (100 $\left.\mu \mathrm{l}\right)$ of plasma sample was mixed with $25 \mu \mathrm{l}$ of internal standard solution (docetaxel, $4 \mu \mathrm{g} / \mathrm{ml}$ in methanol, previously evaporated). After vortex mixing, liquid-liquid extraction was accomplished by adding $4 \mathrm{ml}$ of tert-buthylmethylether following vortex gentle agitation ( $1 \mathrm{~min})$. The mixture was centrifuged for $10 \mathrm{~min}$ at $5000 \mathrm{rpm}$, the organic layer was transferred to a clean vial and evaporated until dry (Savant, Barcelona, Spain). Finally, the residue was dissolved in $125 \mu 1$ of reconstitution solution (acetonitrile $/ 0,01 \mathrm{M}$ phosphate buffer, 50/50 v/v, $\mathrm{pH}=2.0$ ) and transferred to auto-sampler vials, capped and placed in the HPLC auto sampler. A $100 \mu \mathrm{l}$ aliquot of each sample was injected onto the HPLC column.

Under these experimental conditions the run time was $14 \mathrm{~min}$. The limit of quantification was calculated as $80 \mathrm{ng} / \mathrm{ml}$ with a relative standard deviation of $5.2 \%$. Accuracy values during the same day (intra-day assay) at low, medium and high concentrations of PTX was always within the acceptable limits (-1.81 and 3.49\%) at all concentrations tested.

\subsection{Pharmacokinetic data analysis}


The pharmacokinetic analysis of concentration-time data, obtained after the administration of the different PTX formulations, were analyzed using a noncompartimental model using Phoenix WinNonlin 6.0 software (Pharsight Corporation, Mountain View, EEUU). The pharmacokinetic parameters estimated were: the peak of maximun concentration $\left(\mathrm{C}_{\max }\right)$, time to peak concentration $\left(T_{\max }\right)$, the half-life of the terminal phase $\left(t_{1 / 2}\right)$, the area under the concentration-time curve from time 0 to 8 or 24 hours $\left(\mathrm{AUC}_{0-8}, \mathrm{AUC}_{0-24}\right)$, the mean residence time $(\mathrm{MRT})$ and the total body clearance $(\mathrm{Cl})$. The mean residence time $(\mathrm{MRT})$ was calculated as the AUMC (area under the concentration-time curve at the first moment) divided by AUC. The absolute availability (F) was calculated according to:

$$
\mathrm{F}=\mathrm{AUC}_{\text {oral }} / \mathrm{AUC}_{\text {i.v. }}
$$

where $\mathrm{AUC}_{\text {oral }}$ and $\mathrm{AUC}_{\text {i.v. }}$ were the area under the concentration-time curve after the oral and i.v. administration, respectively.

\subsection{Statistical analysis}

The results were expressed as mean values \pm SD. The Mann-Withney U-test was used to investigate statistical differences. In all cases, $\mathrm{p}<0.05$ was considered to be significant. All calculations were performed using $\operatorname{SPSS}^{\circledR}$ statistical software program $\left(\operatorname{SPSS}^{\circledR}\right.$ 14, Microsoft, USA).

\section{Results}

\subsection{Characterization of the micelles}

The main physico-chemical properties of the micelles are presented in Table 1. The resulted stabilized micelles have an average diameter less than $200 \mathrm{~nm}$, narrow distribution and negative zeta-potential values. As shown, drug-loaded micelles did not differ in size 
compared to the non-loaded micelles. The latter is due to the fact that the dense poly-PETA network locks the micellar structure and does not allow any re-arrangement and expansion.

Physical stability of the stabilized micelles was evaluated by observation of their size and polydispersity with time. The size was measured in aqueous media with increasing salt concentration aiming to investigate the influence of the ionic strength. The ratio between the size observed after 24 hours in different media (aqueous dispersion, $0.154 \mathrm{M}$ and $0.308 \mathrm{M}$ $\mathrm{NaCl}$ solution) to initial size measured in distilled water was calculated (Figure 1). Since the size ratios were in a narrow range, the micelles were considered stable.

In order to evaluate the influence of the initial drug/stabilized micelles ratio on drug loading degree, different concentrations of rhodamine/ paclitaxel with respect to the micelle concentration were varied. The results demonstrated that the increase of the initial concentration from 1 to $1.5 \mathrm{mg}$ increased loading degree for both molecules (Figure 2). However, further increase of initial paclitaxel concentration decreased encapsulation efficiency suggesting that the optimal ratio between initial paclitaxel and stabilized micelles was $1: 20$.

\subsection{Gastrointestinal transit of the stabilized micelles}

The study was performed using the stabilized micelles loaded with rhodamine (R-PM) as described above (an initial ratio 1:20). The study included oral administration of micelles and evaluation of the localization of rhodamine in the gastrointestinal mucosa. In parallel, control solution of rhodamine with a concentration similar to the rhodamine concentration into the micelles was administered. The results showed that the total amount of rhodamine within the gastrointestinal mucosa decreased with time (Figure 3). In fact, one hour post administration about $40 \%$ of the given dose was found in the mucosa (mainly in the stomach) whereas, 2 hours later, only $21 \%$ of the rhodamine dose remained within the mucosa. The rhodamine 
dose remained $8 \mathrm{~h}$ post-administration (18\%) was similar to that found $3 \mathrm{~h}$ postadministration, which clearly demonstrated the prolonged transit of micellar rhodamine. In any case, these percentages were significantly higher $(p<0.05)$ than those observed for the control solution of rhodamine (Figure 4).

\subsection{Pharmacokinetic study of paclitaxel when loaded in stabilized Pluronic micelles}

For the pharmacokinetic study, Taxol ${ }^{\circledR}$ and PTX-PM were intravenously or orally administered to mice. Figure 5 illustrates plasma profiles of PTX after i.v. administration of PTX-loaded micelles or Taxol ${ }^{\circledR}$ to mice. As shown, plasma concentrations of both formulations (micelles and commercial) were similar during the first hour postadministration. Then, plasma curve of PTX-PM was characterised by a plateau in the concentration during at least 2 hours followed by a low decrease with time. On the contrary, for Taxol®, the plasma curve was characterised by a rapid decline in the paclitaxel plasma levels during the first $2 \mathrm{~h}$ after administration followed by a slow fall of drug concentrations till the end of the experiment ( 8 hours). Under these circumstances, the micelles provided higher AUC (1.2-fold) compared to Taxol (Table 2). Further, when commercial Taxol ${ }^{\circledR}$ was administered to mice by the oral route, no PTX plasma levels were found (Figure 6). On the contrary, when PTX was included into micelles, these formulations displayed drug levels in plasma for at least $8 \mathrm{~h}$. The plasma curve was characterised by a high $\mathrm{C}_{\max } 30 \mathrm{~min}$ postadministration, only 3-times lower than that obtained with the same formulation i.v. administered (Table 2), followed by a slow and almost constant decline during the following 8 hours. The oral bioavailability of paclitaxel when loaded into the stabilized Pluronic micelles was calculated to be about 0.9 .

\section{Discussion}


Dynamic core-shell micelles of $\mathrm{PEO}_{43} \mathrm{PPO}_{15} \mathrm{PEO}_{43}$ copolymer were stabilized by UV induced crosslinking of tetrafunctional acrylate, PETA, within the micelles according to procedure described elsewhere (Petrov et al., 2008). Pentaerythritol tetraacrylate was applied as cross-linking agent because of its ability to form an interpenetrating network in which the polyether chains are physically entrapped. Previous studies have shown that the micelles obtained with large amount of PETA (10-50 mass\% with respect to PPO fraction) are extremely stable and do not disaggregate upon dilution in water and organic solvents and maintain their integrity even when irradiated with ultrasound (Petrov et al., 2006). In the case of aggregates stabilized with smaller amount of PETA (3 mass \%), copolymer molecules can dissociate from the polymer network resulting in aggregates dissociation (Li et al., 2007). Thus, one may play with the long-term stability of the micelles by tuning the density of polyPETA network. Taking into account the above considerations, the cross-linking agent was used in a concentration of $30 \%$ with respect to PPO fraction. This network does not decrease noticeably the loading capacity of PPO core and, on the other hand, does not influence the flexibility of extended hydrophilic PEO-chains of resulted stabilized micelles. The latter is very important taking into account that the mobility of hydrophilic chains on the periphery of drug carriers determines both the stability and the in vivo distribution of the particles. In this view, our observations showed that the size and polydispersity of the stabilized Pluronic micelles did not change even in the presence of electrolyte. This fact suggested that the PEO chains are extended and prevent any agglomeration of the micelles in the presence of electrolytes.

Two methods have been reported for loading of polymeric micelles with drug molecules, in particular physical and chemical loading (Jones and Leroux, 1999). The second method includes formation of chemical bonds between drug molecule and polymeric carrier, usually during the process of micelles formation. In the present study the first method was applied. 
The pre-stabilized micelles were loaded with fluorescent marker rhodamine B or with paclitaxel by solvent evaporation technique. Comparing both molecules, higher loading and encapsulation efficiency was achieved with paclitaxel. The latter fact was explained with the more hydrophobic nature of paclitaxel $(\log \mathrm{P}$ of 3.5$)$ than rhodamine $(\log \mathrm{P}$ of 1.95$)$.

Gastrointestinal transit of the stabilized micelles was evaluated by oral administration to rats. The most important finding was that the transit of the rhodamine-loaded micelles was prolonged compared to the control solution of free rhodamine. Thus, 8 hours after administration only $6 \%$ of rhodamine applied in the form of solution was spread on the whole GIT versus $18 \%$ for stabilized micelles (Figure 4). Another interesting point was that the micelles were concentrated in the small intestine of animals after oral administration (I3 and I4 portions in Figure 3). All of these results appear to be in line with previous studies about the interaction of micelles with the intestinal mucosa (Gaucher et al., 2010). In fact, the size and hydrophilicity of the shell may limit their interaction with the gut contents and would favour the diffusion of these carriers through the mucus layer. Similar phenomenon has been previously reported for pegylated poly(anhydride) nanoparticles (Yoncheva et al., 2007). The authors postulated that the "brush" layer of PEG-chains reduced an interaction between nanoparticles and mucin, which corresponded to better adhesion and facilitated their proximal contact with enterocytes. In the present study, the extended flexible PEO-chains in the micelle shell probably function in a similar way.

Intravenous administration of Taxol $^{\circledR}$ and PTX-PM into mice showed a significant improvement of pharmacokinetic parameters after administration of micellar paclitaxel (Table 2). More important, the higher MRT (about 5-times) and lower $\mathrm{Cl}$ values (about 2-fold) indicated that PTX-PM formulation increased the time of systemic circulation. This fact could be explained with the protective "stealth" properties of micelle PEO-shell that hinders their recognition and uptake by the cells of RES. These results are in agreement with previous data 
published by Kabanov and collaborators (Kabanov et al., 2002). This manuscript studied the pharmacokinetics and biodistribution of Pluronics after single i.v. administration and reported that these block copolymers remained in circulation a substantial period of time. The results obtained after oral administration of our stabilized micelles were even more interesting. The AUC calculated after oral administration of micelles was similar to that calculated for the i.v. administration of Taxol $^{\circledR}$ (Table 2). On the other hand, the mean residence time of paclitaxel after oral administration of PTX-PM was almost two-times longer than that achieved after i.v. administration of micelles and 9-times longer than Taxol ${ }^{\circledR}$. These data together with the low clearance and high distribution volume (2-fold higher than i.v. Taxol $\left.{ }^{\circledR}\right)$ indicated efficient oral absorption of PTX. In our opinion, the efficient oral absorption of PTX is associated with the properties of the stabilized micelles. Based on the results from the biodistribution studies with rhodamine loaded micelles (R-PM) it could be considered that the flexible PEO-chains in micelles shell enable micelle penetration through the mucus layer, facilitating their transport to the absorptive surface of the enterocytes. On the other hand, it has been described that Pluronics and other non-ionic surfactants inhibit the activity of intestinal Pgp (Batrakova et al., 1998a; 1998b). This fact has been explained by a combination of decreased ATPase activity and ATP depletion due to increased membrane fluidity (Batrakova et al., 2004; Bansal et al., 2009). Nonetheless, studies performed on various cell models have shown that polymeric micelles could be internalized, with fluid-phase pinocytosis appearing as a major route (Allen et al., 1999; Luo et al., 2002). More recently, Mathot et al. have found that micelles obtained from monomethylether poly(ethyleneglycol)(750)-poly(caprolactone-cotrimethylene carbonate) can cross lipid bilayers via passive diffusion and demonstrate an oral bioavailability of $40 \%$ in rats (Mathot et al., 2007). Taking into account these reports, the results in the present study may be explained by an increased absorption of paclitaxel when 
loaded into the newly developed stabilized micelles, an absorption or translocation of micelles through the gastrointestinal mucosa or a combination of both phenomena.

In summary, the present study demonstrated the development of new, in particular stabilized micelles, as paclitaxel delivery system. The extended PEO-chains in the micelle shell probably enabled interactions between epithelial cells of gastrointestinal mucosa and micelles, which in turn prolonged gastric residence time of paclitaxel. In vivo study showed an improvement of pharmacokinetic parameters after intravenous and oral administration of PTX-loaded stabilized Pluronic micelles. Both, the prolonged gastric residence and the high physical stability of micelles achieved by cross-linking of micelle core, have been considered prerequisite for the efficient oral paclitaxel absorption.

\section{Acknowledgements}

This work was supported by the Ministry of Science and Innovation (project SAF200802538), Grant PFIS (2008, Instituto de Salud Carlos III) and Foundation "Caja Navarra: Tú eliges, tú decides" (Nanotecnología y Medicamentos, number 10828) in Spain. P.P. acknowledges the financial support by the National Science Fund of Bulgaria (National Centre for New Materials UNION, contract no. DCVP-02/2).

\section{References}

Agüeros, M., Zabaleta, V., Espuelas, S., Campanero, M.A., Irache, J.M., 2010. Increased oral bioavailability of paclitaxel by its encapsulation through complex formation with cyclodextrins in poly(anhydride) nanoparticles. J. Control. Release 145, 2-8.

Alexandridis, P., Hatton, T.A., 1995. Poly(ethylene oxide)-poly(propylene oxide)poly(ethylene oxide) block copolymer surfactants in aqueous solutions and at interfaces: 
thermodynamics, structure, dynamics, and modeling. Colloids Surf. A: Physicochem. Eng. Aspects 96, 1-46.

Allen, C., Yu, Y., Eisenberg, A., Maysinger, D., 1999. Cellular internalization of PCL(20)-bPEO(44) block copolymer micelles. Biochim. Biophys. Acta 1421, 32-38.

Arbos, P., Arangoa, M.A., Campanero, M.A., Irache, J.M., 2002. Quantification of the bioadhesive properties of protein-coated PVM/MA nanoparticles. Int. J. Pharm. 242, 129-136. Bansal, T., Akhtar, N., Jaggi, M., Khar, R.K., Talegaonkar, S., 2009. Novel formulation approaches for optimising delivery of anticancer drugs based on P-glycoprotein modulation. Drug Discovery Today 14, 1067-1074.

Batrakova, E.V., Han, H.Y., Alakhov, V., Miller, D.W., Kabanov, A.V., 1998a. Effects of pluronic block copolymers on drug absorption in Caco-2 cell monolayers. Pharm. Res. 15, $850-855$

Batrakova, E.V., Han, H.Y., Miller, D.W., Kabanov, A.V., 1998b. Effects of pluronic P85 unimers and micelles on drug permeability in polarized BBMEC and Caco-2 cells. Pharm. Res. $15,1525-1532$.

Batrakova, E.V., Li, S., Li, Y., Alakhov, V.Y., Kabanov, A.V., 2004. Effect of pluronic P85 on ATPase activity of drug efflux transporters. Pharm. Res. 21, 2226-2233.

Batrakova, E.V., Kabanov, A.V., 2008. Pluronic block copolymers: evolution of drug delivery concept from inert nanocarriers to biological response modifiers. J. Control. Release 130, 98-106.

Boddy, A.V., Plummer, E.R., Todd, R., Sludden, J., Griffin, M., Robson, L., Cassidy, J., Bissett, D., Bernareggi, A., Verrill, M., Calvert, A., 2005. A Phase I and pharmacokinetic study of paclitaxel poliglumex (Xyotax), investigating both 3-weekly and 2-weekly schedules. Clin. Cancer Res. 11, 7834-7840. 
Chen, Q., Zhang, Q.Z., Liu, J., Li, L.Q., Zhao, W.H., Wang, Y.J., Zhou, Q.H., Li, L., 2003. Multi-center prospective randomized trial on paclitaxel liposome and traditional taxol in the treatment of breast cancer and non-small-cell lung cancer. Chin. J. Oncol. 25, 190-192.

Forrest, M.L., Yanez, J.A., Remsber, C.M., Ohgami, Y., Kwon, G.S., Davies, N.M., 2008. Paclitaxel prodrugs with sustained release and high solubility in poly(ethylene glycol-b$\operatorname{poly}(\varepsilon$-caprolactone) micelle nanocarriers: pharmacokinetic disposition, tolerability, and cytotoxicity. Pharm. Res. 25, 194-206.

Gaucher, G., Satturwar, P., Jones, M.C., Furtos, A., Leroux, J.C., 2010. Polymeric micelles for oral drug delivery. Eur. J. Pharm. Biopharm. 76, 147-158.

Gregory, R.E., DeLisa, A.F., 1993. Paclitaxel: a new antineoplastic agent for refractory ovarian cancer. Clin. Pharm. 12, 401-415.

Hamaguchi, T., Matsumura, Y., Suzuki, M., Shimizu, K., Goda, R., Nakamura, I., Nakatomi, I., Yokoyama, M., Kataoka, K., Kakizoe, T., 2005. NK105, a paclitaxel-incorporating micellar nanoparticle formulation, can extend in vivo antitumour activity and reduce the neurotoxicity of paclitaxel. Br. J. Cancer 92, 1240-1246.

Han, L.M., Guo, J., Zhang, L.J., Wang, Q.S., Fang, X.L., 2006. Pharmacokinetics and biodistribution of polymeric micelles of paclitaxel with Pluronic P123. Acta Pharmacol. Sin. $27,747-753$.

Huang, J., Si, L., Jiang, L., Fan, Z., Qiu, J., Li, G., 2008. Effect of pluronic F68 block copolymer on P-glycoprotein transport and CYP3A4 metabolism. Int. J. Pharm. 356, 351-353. Jones, M.C., Leroux, J.C., 1999. Polymeric micelles - a new generation of colloidal drug carriers. Eur. J. Pharm. Biopharm. 48, 101-111.

Kabanov, A.V., Batrakova, E.V., Alakhov, V.Y., 2002. Pluronic block copolymers for overcoming drug resistance in cancer. Adv. Drug Deliv. Rev. 54, 759-779. 
Kabanov, A.V., Batrakova, E.V., Alakhov, V.J., 2002. Pluronic block copolymers as novel polymer therapeutics for drug and gene delivery. J. Control. Release 82, 189-212.

Koziara, J., Whisman, T., Tseng, M., Mumper, R.J., 2006. In-vivo efficacy of novel paclitaxel nanoparticles in paclitaxel-resistant human colorectal tumors. J. Control. Release 112, 312319.

Letchford, K., Liggins, R., Wasan, K.M., Burt, H., 2009. In vitro human plasma properties of poly(ethylene glycol)-block-poly(caprolactone) nanoparticles. Eur. J. Pharm. Biopharm. 71, 196-206.

Li, F., Ketelaar, T., Marcelis, A.T.M., Leermakers, F.A.M., Cohen Stuart, M,A,, Sudhölter, E.J.R., 2007. Stabilization of polymersome vesicles by an interpenetrating polymer network. Macromolecules 40, 329-333.

Luo, L., Tam, J., Maysinger, D., Eisenberg, A., 2002. Cellular internalization of poly(ethylene oxide)-b-poly(epsilon-caprolactone) diblock copolymer micelles. Bioconjug. Chem. 13, 12591265.

Maeda, H., Wu, J., Sawa, T., Matsumura, Y., Hori, K., 2000. Tumor vascular permeability and the EPR effect in macromolecular therapeutics: a review. J. Control. Release 65, 271-284. Malingre, M.M., Beijnen, J.H., Schellens, J.H., 2001. Oral delivery of taxanes. Invest. New Drugs $19,155-162$.

Mathot, F., des Rieux, A., Ariën, A., Schneider, Y.J., Brewster, M., Préat, V., 2007. Transport mechanism of mmePEG750P(CL-co-TMC) polymeric micelles across the intestinal barrier. J. Control. Release 124, 134-43.

Onetto, N., Canetta, R., Winograd, B., Catane, R., Dougan, M., Grechko, J., Burroughs, J., Rozencweig, M., 1993. Overview of Taxol safety. J Natl. Cancer Inst. Monogr. 15, 131-39. Petrov, P., Bozukov, M., Burkhardt, M., Muthukrishnan, S., Mueller, A.H.E., Tsvetanov, Ch., 2006. Stabilization of polymeric micelles with a mixed poly(ethylene oxide)/poly(2- 
hydroxyethyl methacrylate) shell by formation of poly(pentaerythritol tetraacrylate) nanonetworks within the micelles. J. Mater. Chem. 16, 2192-2199.

Petrov, P., Jiayin, Y., Yoncheva, K., Müller, A., Tsvetanov, Ch., 2008. Wormlike morphology formation and stabilization of "Pluronic P123" micelles by solubilization of pentaerythritol tetraacrylate. J. Phys. Chem. B 112, 8879-8883.

Rapoport, N., 1999. Stabilization and activation of Pluronic micelles for tumor-targeted drug delivery. Colloids Surf. B: Biointerfaces 16, 93-111.

Roy, V., LaPlant, B.R., Gross, G.G., Bane, C.L., Palmieri, F.M., 2009. Phase II trial of weekly nab (nanoparticle albumin-bound)-paclitaxel (nab-paclitaxel) (Abraxane) in combination with gemcitabine in patients with metastatic breast cancer (N0531). Ann. Oncol. 20, 449-453.

Singla, A.K., Garg, A., Aggarwal, D., 2002. Paclitaxel and its formulations. Int. J. Pharm. $235,179-192$.

Sparreboom, A., van Zuylen, L., Brouwer, E., Loos, W., de Bruijn, P., Gelderblom, H., Pillay, M., Nooter, K., Stoter, G., Verweij, J., 1999. Cremophor EL-mediated alteration of paclitaxel distribution inhumanblood: clinical pharmacokinetic implications. Cancer Res. 59, 14541457.

Thomas, H., Coley, H.M., 2003. Overcoming multidrug resistance in cancer: an update on the clinical strategy of inhibiting p-glycoprotein. Cancer Control 10, 159-165.

Torchilin, V.P., 2004. Targeted polymeric micelles for delivery of poorly soluble drugs. Cell Mol. Life Sci. 61, 2549-2559.

Wang, X., Zheng, H., Zhu, Z., Wei, Y., Chen, L., 2010. Clinical pharmacokinetics of paclitaxel liposome with a new route of administration in human based on the analysis with ultra performance liquid chromatography. J. Pharm. Sci. 99, 4746-4752. 
Win, K.Y., Feng, S.S., 2006. In vitro and in vivo studies on vitamin E TPGS-emulsified poly(D,L-lactic-co-glycolic acid) nanoparticles for paclitaxel formulation. Biomaterials 27, $2285-2291$

Yoncheva, K., Guembe, L., Campanero, M.A., Irache, J.M., 2007. Evaluation of bioadhesive potential and intestinal transport of pegylated poly(anhydride) nanoparticles. Int. J. Pharm. $334,156-165$.

Zhao, L., Ye, Y., Li, J., Wei, Y.M., 2011. Preparation and the in vivo evaluation of paclitaxel liposomes for lung targeting delivery in dogs. J. Pharm. Pharmacol. 63, 80-86. 
Table 1. Physico-chemical properties of non-loaded stabilized Pluronic micelles (PM), rhodamine-loaded (R-PM) and paclitaxel-loaded micelles (PTX-PM). Data expressed as the mean $\pm \mathrm{SD}, \mathrm{n}=3$.

\begin{tabular}{cccc}
\hline Sample & Size, nm & PI & Zeta-potential, mV \\
\hline PM & $177.2 \pm 8$ & 0.252 & $-12.4 \pm 3$ \\
R-PM & $165.4 \pm 16$ & 0.372 & $-17.2 \pm 3$ \\
PTX-PM & $180.0 \pm 5$ & 0.235 & $-15.2 \pm 2$ \\
\hline
\end{tabular}


Table2. Plasma pharmacokinetic parameters (mean $\pm \mathrm{SD}$ ) of polymeric micelles in $\mathrm{C} 57 \mathrm{BL} / 6 \mathrm{~J}$ female mice following a single i.v. and oral administration. Dose PXL $=10 \mathrm{mg} / \mathrm{kg}$.

\begin{tabular}{|c|c|c|c|c|c|c|c|c|c|}
\hline Sample & Route & $\begin{array}{c}\text { AUC } \\
(\mu \mathrm{g} \mathrm{h} / \mathrm{mL})\end{array}$ & $\begin{array}{l}\mathbf{C}_{\max } \\
(\mathrm{ng})\end{array}$ & $\begin{array}{c}\mathbf{T} \\
\max \\
(\mathrm{h})\end{array}$ & $\begin{array}{l}\mathbf{T}_{1 / 2} \mathbf{z} \\
\left(\mathrm{h}^{-1}\right)\end{array}$ & $\begin{array}{c}\text { MRT } \\
(\mathrm{h})\end{array}$ & $\begin{array}{c}\mathbf{V} \\
(\mathrm{mL})\end{array}$ & $\begin{array}{c}\mathbf{C l} \\
(\mathrm{mL} / \mathrm{h})\end{array}$ & $\mathbf{F}$ \\
\hline Taxol $^{\circledR}$ & i.v. & $90.2 \pm 24.5$ & $60.5 \pm 6.4$ & 0 & $2.27 \pm 0.5$ & $1.39 \pm 0.4$ & $5.8 \pm 0.6$ & $2.7 \pm 0.3$ & \\
\hline PTX-PM & i.v. & $110.0 \pm 29.1$ & $68.1 \pm 3.8$ & 0 & $2.2 \pm 0.1$ & $5.1 \pm 2.4$ & $6.3 \pm 0.6$ & $1.3 \pm 0.8$ & \\
\hline Taxol $^{\circledR}$ & Oral & ND & ND & ND & ND & ND & ND & ND & ND \\
\hline PTX-PM & Oral & $99.2 \pm 17.3$ & $19.2 \pm 3.6$ & 0.5 & $0.1 \pm 0.0$ & $12.7 \pm 1.6$ & $12.3 \pm 1.5$ & $1.1 \pm 0.3$ & 0.9 \\
\hline
\end{tabular}

AUC area under the concentration-time curve from time 0 to $8 ; \mathrm{C}_{\max }$ : peak concentration; $\mathrm{T}_{\max }$ : time to peak concentration; MRT: mean residence time; $\mathrm{t}_{1 / 2 \mathrm{z}}$ : half-life of the terminal phase. F: oral bioavailability. 


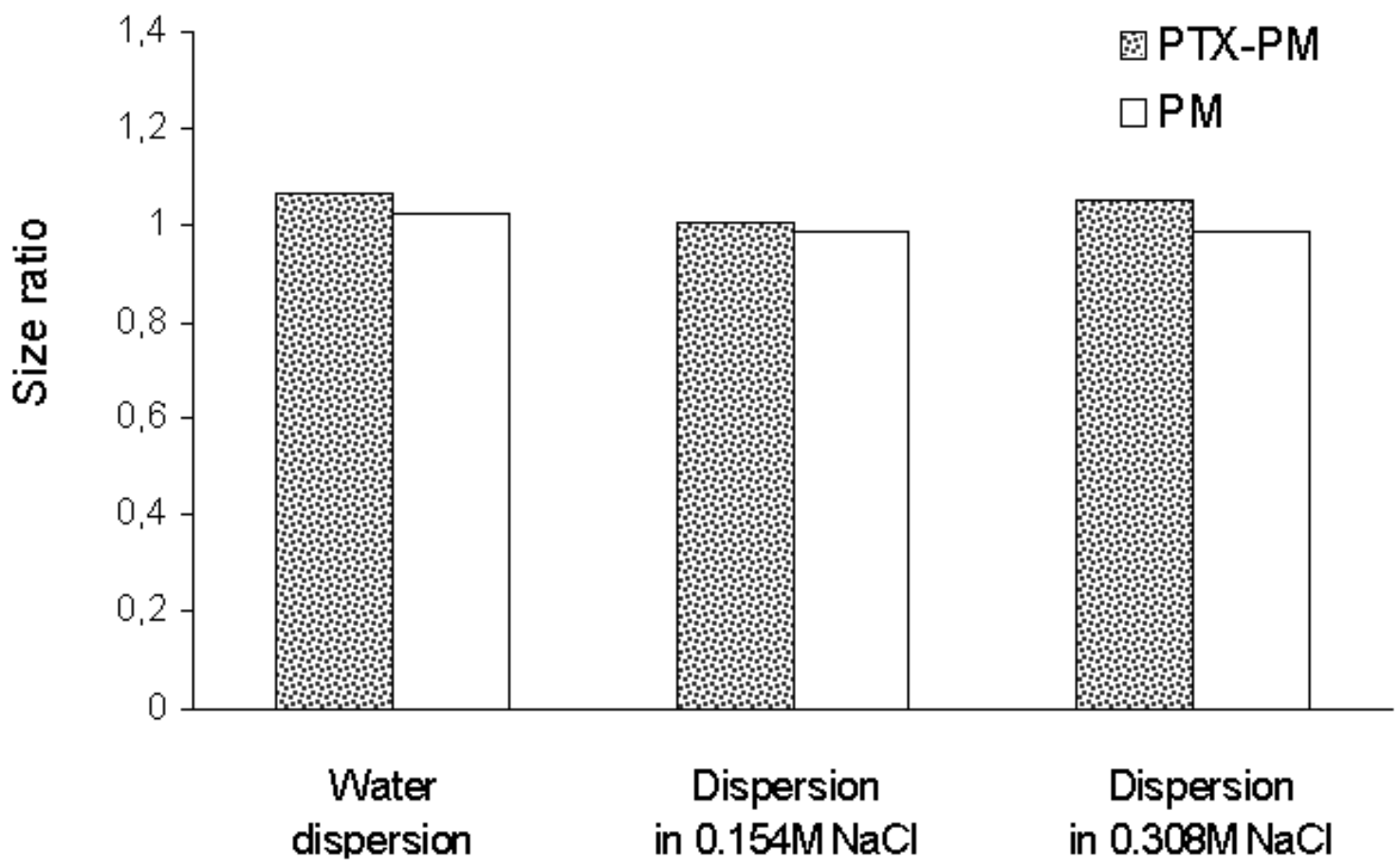

Figure 1. Chart diagrams representing the ratio between the size of the micelles observed after $24 \mathrm{~h}$ incubation in different media to initial size measured in distilled water. 

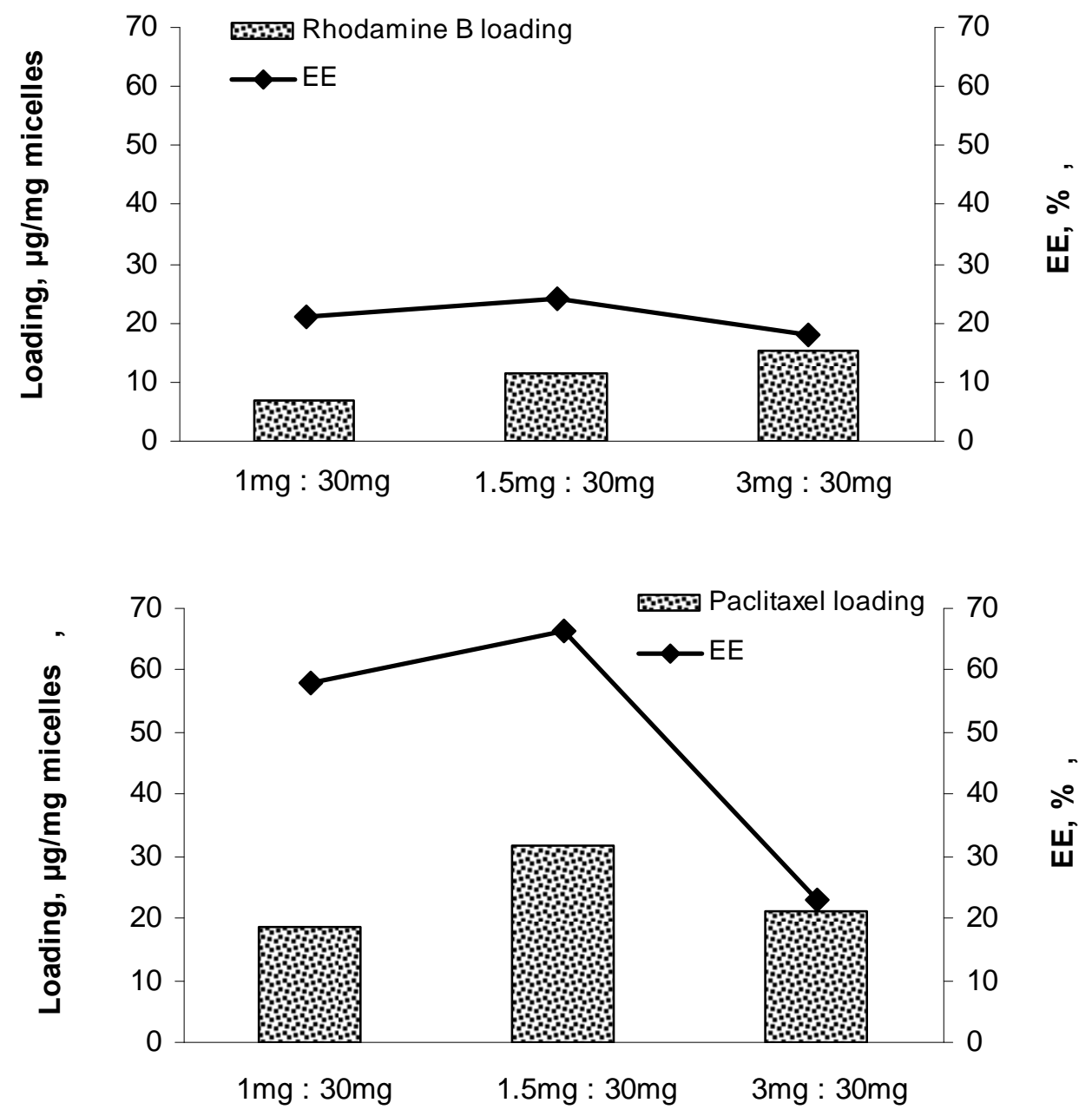

Figure 2. Influence of the initial concentration of rhodamine (a) or paclitaxel (b) on the loading degree into micelles and encapsulation efficiency. Data expressed as the mean $\pm \mathrm{SD}$, $\mathrm{n}=3$. 


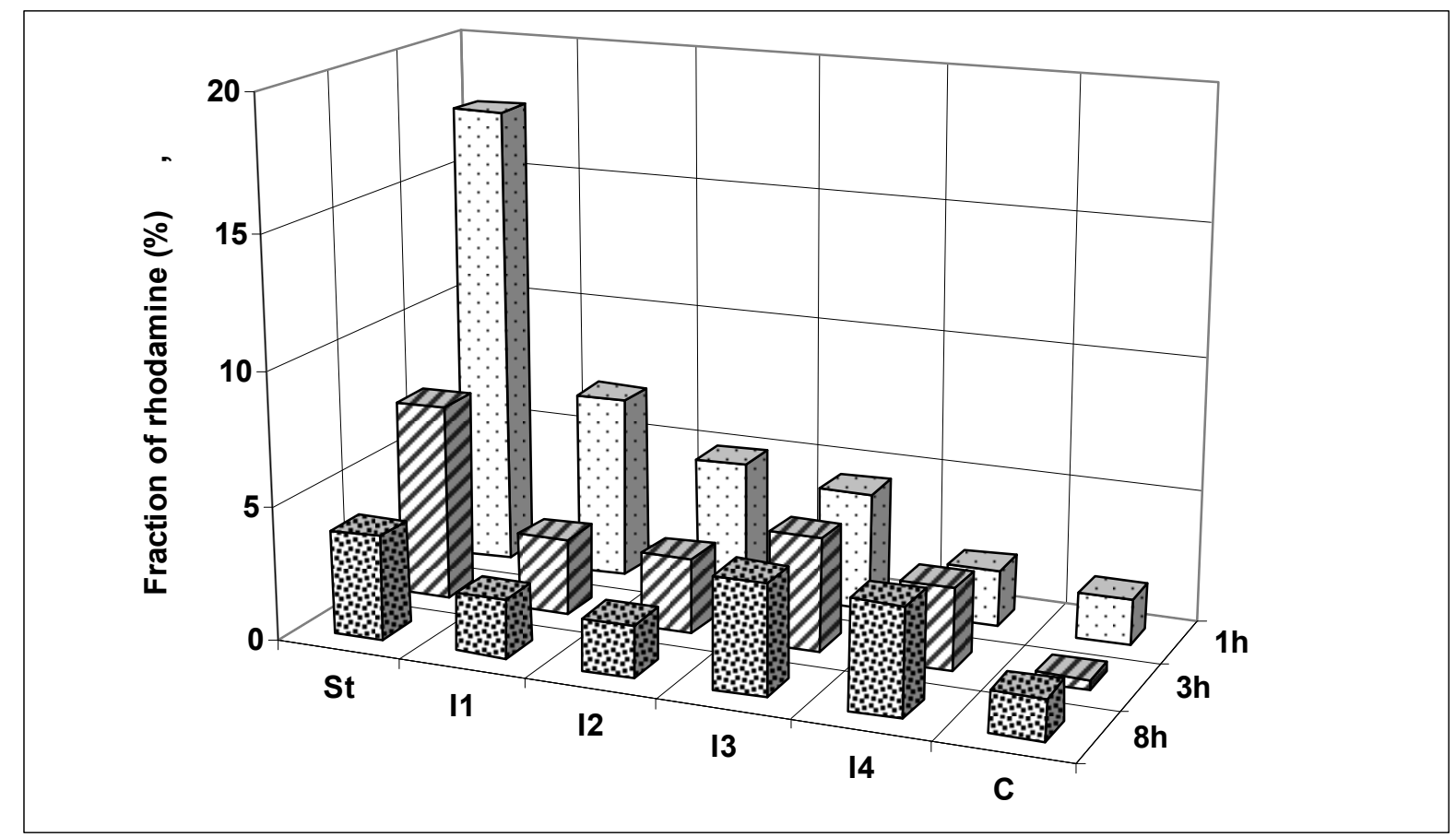

Figure 3. Distribution of the rhodamine in the gastrointestinal segments after oral administration of rhodamine-loaded stabilized Pluronic micelles (R-PM). St-stomach, intestinal parts: I1 - duodenum, I2 and I3 - jejunum, I4 representing ileum, C-caecum). 


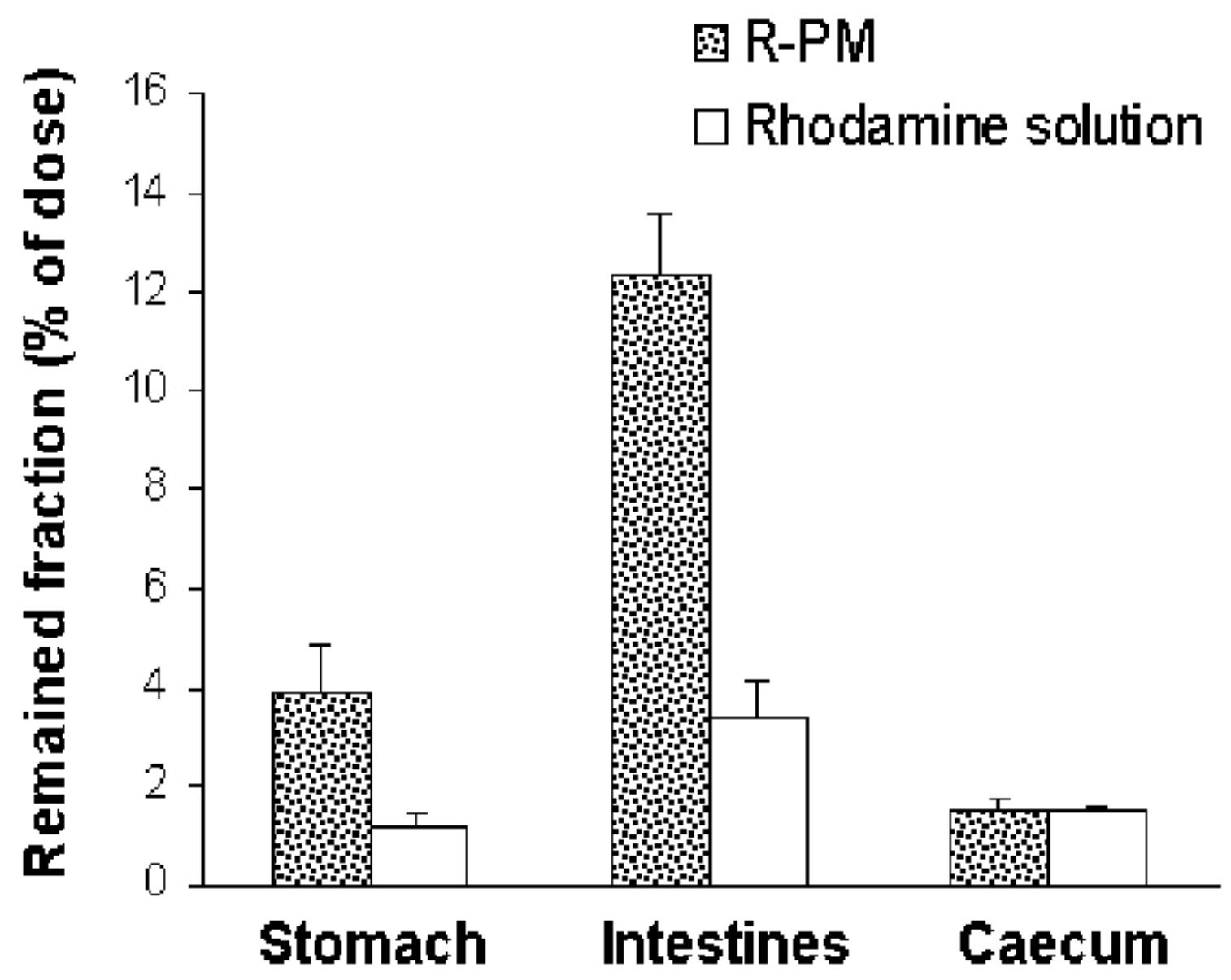

Figure 4. Remained rhodamine fractions in the gastrointestinal segments of rats 8 hours after oral administration of rhodamine-loaded stabilized micelles and control rhodamine solution. 


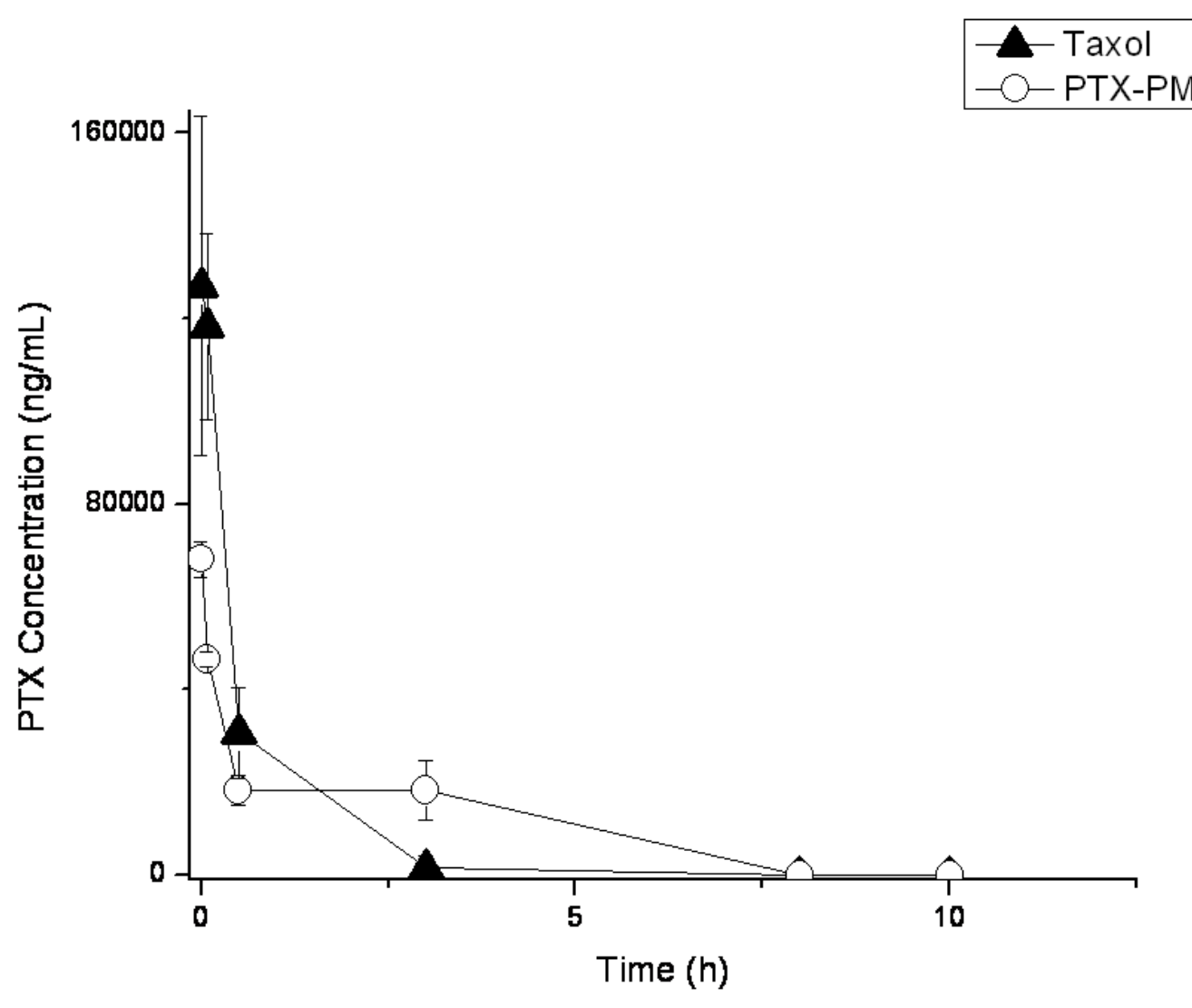

Figure 5. Paclitaxel plasmatic levels after intravenous administration (single dose of $10 \mathrm{mg} / \mathrm{kg}$ ) of either stabilized paclitaxel-loaded micelles (PTX-PM) or Taxol ${ }^{\circledR}$. Data expressed as mean $\pm \operatorname{SD}(n=5)$. 


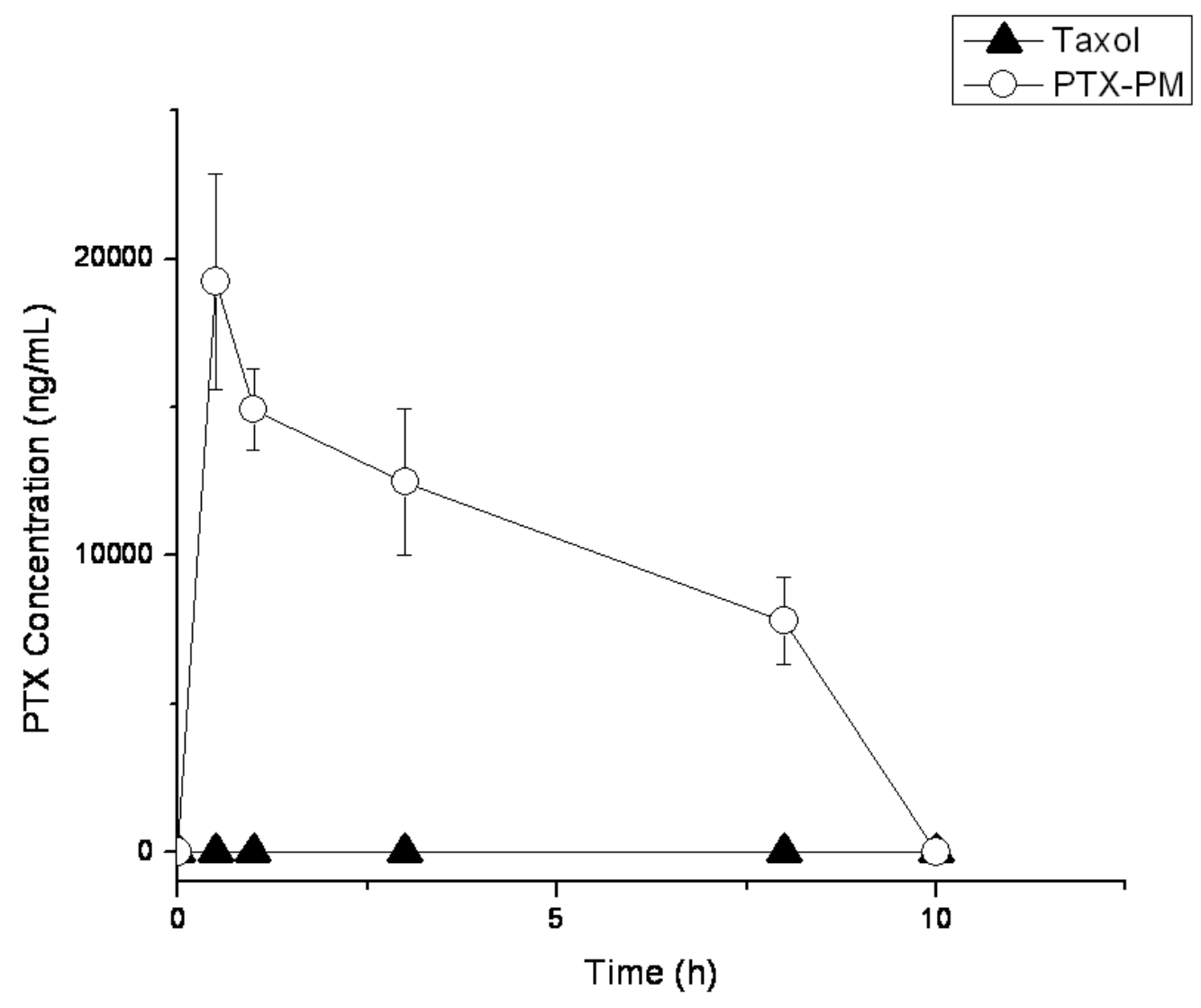

Figure 6. Paclitaxel plasmatic levels after oral administration (single dose of $10 \mathrm{mg} / \mathrm{kg}$ ) of either stabilized paclitaxel-loaded micelles (PTX-PM) or Taxol ${ }^{\circledR}$. Data expressed as mean \pm $\operatorname{SD}(n=5)$. 